\title{
The $\left(G^{\prime} / G\right)$-Expansion Method for Abundant Traveling Wave Solutions of Caudrey-Dodd-Gibbon Equation
}

\author{
Hasibun Naher, Farah Aini Abdullah, and M. Ali Akbar \\ School of Mathematical Sciences, Universiti Sains Malaysia, 11800 Penang, Malaysia \\ Correspondence should be addressed to Hasibun Naher, hasibun06tasauf@gmail.com \\ Received 24 June 2011; Accepted 22 September 2011 \\ Academic Editor: Kue-Hong Chen \\ Copyright (c) 2011 Hasibun Naher et al. This is an open access article distributed under the \\ Creative Commons Attribution License, which permits unrestricted use, distribution, and \\ reproduction in any medium, provided the original work is properly cited. \\ We construct the traveling wave solutions of the fifth-order Caudrey-Dodd-Gibbon (CDG) equa- \\ tion by the $\left(G^{\prime} / G\right)$-expansion method. Abundant traveling wave solutions with arbitrary parame- \\ ters are successfully obtained by this method and the wave solutions are expressed in terms of the \\ hyperbolic, the trigonometric, and the rational functions. It is shown that the $\left(G^{\prime} / G\right)$-expansion \\ method is a powerful and concise mathematical tool for solving nonlinear partial differential \\ equations.
}

\section{Introduction}

The investigation of exact traveling wave solutions of nonlinear partial differential equations (NLPDEs) plays an important role in the analysis of complex physical phenomena. The NLPDEs appear in physical sciences, various scientific and engineering problems, such as, fluid mechanics, plasma physics, optical fibers, biology, solid state physics, chemical kinematics, chemical physics, chemistry and many others. In recent years, to obtain exact traveling wave solutions of NLPDEs, many effective and powerful methods have been presented in the literature, such as the Backlund transformation [1], the tanh function method [2], the extended tanh function method [3], the variational iteration method [4], the Adomian decomposition method $[5,6]$, the homotopy perturbation method [7], the F-expansion method [8], the Hirota's bilinear method [9], the exp-function method [10], the Cole-Hopf transformation [11], the general projective Riccati equation method [12] and others [13-20], Nowaday, searching analytical solutions of the NLPDEs has become more lucrative partly due to the accessibility computer symbolic systems, like Maple, Mathematica, 
and Matlab, which help us to calculate the complicated and wearisome algebraic calculations on computer.

Recently, Wang et al. [21] introduced a new direct method called the $\left(G^{\prime} / G\right)$-expansion method to seek traveling wave solutions of the nonlinear evolution equations. Abazari [22] implemented the $\left(G^{\prime} / G\right)$-expansion method to NLEEs related to fluid mechanics. Zheng [23] used the method for getting exact traveling wave solutions of two different types of equations. Feng et al. [24] used the method to seek solutions of the Kolmogorov-PetrovskiiPiskunov equation. Liu et al. [25] concerned the method to simplified $\mathrm{MCH}$ equation and Gardner equation. Feng [26] applied the method to the seventh-order Sawada-Kotera equation.

At present time, Guo and Zhou [27] first proposed the extended $\left(G^{\prime} / G\right)$-expansion method based on new ansatz. They applied the method to the Whitham-Broer-Kaup-Like equations and couple Hirota-Satsuma KdV equations. Zayed et al. [28] used extended $\left(G^{\prime} / G\right)$-expansion method to some nonlinear PDEs in mathematical physics. Zhang et al. [29] presented an improved $\left(G^{\prime} / G\right)$-expansion method for solving nonlinear evolution equations (NLEEs). Li et al. [30] introduced $\left(G^{\prime} / G, 1 / G\right)$-expansion method to obtain traveling wave solutions of the Zakharov equations. Hayek [31] proposed extended $\left(G^{\prime} / G\right)$-expansion method for constructing exact solutions to the KdV Burgers equations with power-law nonlinearity.

In this paper, our aim is to investigate abundant new exact traveling wave solutions of the fifth-order Caudrey-Dodd-Gibbon equation by the $\left(G^{\prime} / G\right)$-expansion method.

\section{Description of the $\left(G^{\prime} / G\right)$-Expansion Method}

Suppose that the nonlinear partial differential equation is of the form

$$
P\left(u, u_{t}, u_{x}, u_{t t}, u_{x t}, u_{x x}, \ldots\right)=0,
$$

where $u=u(x, t)$ is an unknown function and $P$ is a polynomial in $u=u(x, t)$ which has various partial derivatives, and the highest order derivatives and nonlinear terms are involved.

The main steps of the $\left(G^{\prime} / G\right)$-expansion method [21] are conveyed in the following.

Step 1. The traveling wave transformation:

$$
u(x, t)=v(\xi), \quad \xi=x+s t,
$$

where $s$ is the wave speed, $\xi$ is the combination of two independent variables $x$ and $t$, transform (2.1) into an ordinary differential equation for $v(\xi)$ :

$$
Q\left(v, s v^{\prime}, v^{\prime}, s^{2} v^{\prime \prime}, s v^{\prime \prime}, v^{\prime \prime}, \ldots\right)=0
$$

where primes denote the ordinary derivatives with respect to $\xi$.

Step 2. If possible, integrate (2.3) term by term for one or more times, yields constant(s) of integration. For simplicity, the integration constant(s) may be zero. 
Step 3. Suppose that the solution of (2.3) can be expressed by a polynomial in $\left(G^{\prime} / G\right)$ :

$$
v(\xi)=\alpha_{m}\left(\frac{G^{\prime}}{G}\right)^{m}+\alpha_{m-1}\left(\frac{G^{\prime}}{G}\right)^{m-1}+\cdots+\alpha_{0}
$$

where $\alpha_{n}(n=0,1,2,3, \ldots, m)$ are constants and $\alpha_{m} \neq 0$, and $G=G(\xi)$ satisfies the second order linear ordinary differential equation (LODE):

$$
G^{\prime \prime}+\lambda G^{\prime}+\mu G=0,
$$

where $\lambda$ and $\mu$ are arbitrary constants. The positive integer $m$ can be determined by considering the homogeneous balance between the highest order derivatives and the nonlinear terms appearing in (2.3).

Step 4. Substituting (2.4) together with (2.5) into (2.3) yields an algebraic equation involving powers of $\left(G^{\prime} / G\right)$. Then, equating coefficients of each power of $\left(G^{\prime} / G\right)$ to zero yields a set of algebraic equations for $\alpha_{n}(n=0,1,2,3, \ldots, m), s, \lambda$, and $\mu$. Since the general solution of (2.5) is known for us, then substituting $\alpha_{n}(n=0,1,2,3, \ldots, m)$ and $s$ together with general solution of (2.5) into (2.4), we obtain exact traveling wave solutions of the nonlinear partial differential equation (2.1).

\section{Application of the Method}

In this section, we apply the method to construct the hyperbolic, the trigonometric, and the rational function solutions of the fifth-order Caudrey-Dodd-Gibbon equation, and the solutions are shown in graphs.

\subsection{The Caudrey-Dodd-Gibbon Equation}

We consider the fifth-order Caudrey-Dodd-Gibbon equation:

$$
u_{t}+u_{x x x x x}+30 u u_{x x x}+30 u_{x} u_{x x}+180 u^{2} u_{x}=0
$$

Now, we use the transformation equation (2.2) into (3.1), which yields,

$$
s v^{\prime}+v^{(5)}+30 v v^{\prime \prime \prime}+30 v^{\prime} v^{\prime \prime}+180 v^{2} v^{\prime}=0,
$$

where primes denote the derivative with respect to $\xi$.

Equation. (3.2) is integrable, therefore, integrating once with respect to $\xi$ yields

$$
s v+v^{(4)}+30 v v^{\prime \prime}+60 v^{3}+C=0,
$$

where $C$ is an integral constant, that is, to be determined later. 
Considering the homogeneous balance between $v^{(4)}$ and $v^{3}$ in (3.3), we obtain $m=2$. Therefore, the solution of (3.3) is of the form:

$$
v(\xi)=\alpha_{2}\left(\frac{G^{\prime}}{G}\right)^{2}+\alpha_{1}\left(\frac{G^{\prime}}{G}\right)+\alpha_{0}
$$

where $\alpha_{2}, \alpha_{1}$, and $\alpha_{0}$ are constants and $\alpha_{2} \neq 0$. Substituting (3.4) together with (2.5) into (3.3) and collecting all terms with the same power of $\left(G^{\prime} / G\right)$ and setting each coefficient of the resulted polynomial to zero, we obtain a set of algebraic equations for $\alpha_{2}, \alpha_{1}, \alpha_{0}, s, \lambda, \mu$, and $C$ as follows:

$$
\begin{aligned}
& 60 \alpha_{2}^{3}+120 \alpha_{2}+180 \alpha_{2}^{2}=0, \\
& 180 \alpha_{2}^{2} \alpha_{1}+240 \alpha_{2} \alpha_{1}+300 \alpha_{2}^{2} \lambda+336 \alpha_{2} \lambda+24 \alpha_{1}=0, \\
& 180 \alpha_{2} \alpha_{1}^{2}+180 \alpha_{2}^{2} \alpha_{0}+240 \alpha_{2}^{2} \mu+120 \alpha_{2}^{2} \lambda^{2}+180 \alpha_{0} \alpha_{2}+330 \alpha_{2} \lambda^{2} \\
& \quad+240 \alpha_{2} \mu+60 \alpha_{1} \lambda+60 \alpha_{1}^{2}+390 \alpha_{2} \alpha_{1} \lambda=0, \\
& 360 \alpha_{2} \alpha_{1} \alpha_{0}+60 \alpha_{1}^{3}+440 \alpha_{2} \lambda \mu+90 \alpha_{1}^{2} \lambda+60 \alpha_{0} \alpha_{1}+40 \alpha_{1} \mu+130 \alpha_{2} \lambda^{3} \\
& \quad+50 \alpha_{1} \lambda^{2}+180 \alpha_{2}^{2} \lambda \mu+300 \alpha_{2} \alpha_{1} \mu+150 \alpha_{2} \alpha_{1} \lambda^{2}+300 \alpha_{0} \alpha_{2} \lambda=0, \\
& 180 \alpha_{2} \alpha_{0}^{2}+180 \alpha_{1}^{2} \alpha_{0}+210 \alpha_{2} \alpha_{1} \lambda \mu+60 \alpha_{2}^{2} \mu^{2}+60 \alpha_{1}^{2} \mu+30 \alpha_{1}^{2} \lambda^{2}+5 \alpha_{2}+136 \alpha_{2} \mu^{2} \\
& \quad+16 \alpha_{2} \lambda^{4}+15 \alpha_{1} \lambda^{3}+60 \alpha_{1} \lambda \mu+232 \alpha_{2} \lambda^{2} \mu+240 \alpha_{0} \alpha_{2} \mu+90 \alpha_{0} \alpha_{1} \lambda+120 \alpha_{0} \alpha_{2} \lambda^{2}=0, \\
& 180 \alpha_{1} \alpha_{0}^{2}+180 \alpha_{0} \alpha_{2} \lambda \mu+16 \alpha_{1} \mu^{2}+5 \alpha_{1}+\alpha_{1} \lambda^{4}+22 \alpha_{1} \lambda^{2} \mu+120 \alpha_{2} \lambda \mu^{2}+30 \alpha_{2} \lambda^{3} \mu \\
& \quad+60 \alpha_{1} \alpha_{2} \mu^{2}+30 \alpha_{1}^{2} \lambda \mu+60 \alpha_{0} \alpha_{1} \mu+30 \alpha_{0} \alpha_{1} \lambda^{2}=0, \\
& 60 \alpha_{0}^{3}+14 \alpha_{2} \lambda^{2} \mu^{2}+\alpha_{1} \lambda^{3} \mu+8 \alpha_{1} \lambda \mu^{2}+16 \alpha_{2} \mu^{3}+C+60 \alpha_{0} \alpha_{2} \mu^{2}+5 \alpha_{0}+30 \alpha_{0} \alpha_{1} \lambda \mu=0
\end{aligned}
$$

Solving the system of algebraic equations with the aid of Maple 13, we obtain two different sets of solution.

Case 1. One has

$$
\begin{gathered}
\alpha_{0}=\frac{-1}{6} \lambda^{2}-\frac{4}{3} \mu, \quad \alpha_{1}=-2 \lambda, \quad \alpha_{2}=-2, \quad s=-\lambda^{4}+8 \mu \lambda^{2}-16 \mu^{2}, \\
C=\frac{1}{9} \lambda^{6}-\frac{4}{3} \lambda^{4} \mu+\frac{16}{3} \lambda^{2} \mu^{2}-\frac{64}{9} \mu^{3},
\end{gathered}
$$

where $\lambda$ and $\mu$ are arbitrary constants.

Case 2. One has

$$
\begin{aligned}
& \alpha_{1}=-\lambda, \quad \alpha_{2}=-1, \quad s=-180 \alpha_{0}^{2}-30 \lambda^{2} \alpha_{0}-22 \mu \lambda^{2}-76 \mu^{2}-\lambda^{4}-240 \mu \alpha_{0}, \\
& C=120 \alpha_{0}^{3}+22 \lambda^{2} \mu^{2}+\lambda^{4} \mu+16 \mu^{3}+136 \alpha_{0} \mu^{2}+30 \lambda^{2} \alpha_{0}^{2}+52 \alpha_{0} \lambda^{2} \mu+\alpha_{0} \lambda^{4}+240 \mu \alpha_{0}^{2},
\end{aligned}
$$

where $\alpha_{0}, \lambda$, and $\mu$ are arbitrary constants. 
Case 1. Substituting (3.6) into (3.4) yields

$$
v(\xi)=-2\left(\frac{G^{\prime}}{G}\right)^{2}-2 \lambda\left(\frac{G^{\prime}}{G}\right)-\frac{1}{6} \lambda^{2}-\frac{4}{3} \mu
$$

Substituting the general solution of (2.5) into (3.8), we obtain three types of traveling wave solutions of (3.3) as follows.

\section{Hyperbolic Function Solutions}

When $\lambda^{2}-4 \mu>0$, substituting the general solution of (2.5) into (3.8), we obtain the following traveling wave solution of (3.3):

$$
v(\xi)=-\frac{\lambda^{2}-4 \mu}{2}\left(\frac{C_{1} \sinh (1 / 2) \sqrt{\lambda^{2}-4 \mu} \xi+C_{2} \cosh (1 / 2) \sqrt{\lambda^{2}-4 \mu} \xi}{C_{1} \cosh (1 / 2) \sqrt{\lambda^{2}-4 \mu} \xi+C_{2} \sinh (1 / 2) \sqrt{\lambda^{2}-4 \mu} \xi}\right)^{2}+\frac{1}{3} \lambda^{2}-\frac{4}{3} \mu,
$$

where $\xi=x+\left(-\lambda^{4}+8 \mu \lambda^{2}-16 \mu^{2}\right) t, C_{1}$, and $C_{2}$ are arbitrary constants. The various known results can be rediscovered, if $C_{1}$ and $C_{2}$ are taken as special values. For example:

(i) if $C_{1}=0$ but $C_{2} \neq 0$, we obtain

$$
v(\xi)=-\frac{\lambda^{2}-4 \mu}{2} \operatorname{coth}^{2} \frac{1}{2} \sqrt{\lambda^{2}-4 \mu} \xi+\frac{1}{3} \lambda^{2}-\frac{4}{3} \mu .
$$

(ii) If $C_{2}=0$ but $C_{1} \neq 0$, we obtain

$$
v(\xi)=-\frac{\lambda^{2}-4 \mu}{2} \tanh ^{2} \frac{1}{2} \sqrt{\lambda^{2}-4 \mu} \xi+\frac{1}{3} \lambda^{2}-\frac{4}{3} \mu .
$$

(iii) If $C_{1} \neq 0$ and $C_{1}^{2}>C_{2}^{2}$, we obtain

$$
v(\xi)=\frac{\lambda^{2}-4 \mu}{2} \operatorname{sech}^{2}\left(\frac{1}{2} \sqrt{\lambda^{2}-4 \mu} \xi+\xi_{0}\right)-\frac{\lambda^{2}}{6}+\frac{2}{3} \mu,
$$

where $\xi_{0}=\tanh ^{-1}\left(C_{2} / C_{1}\right)$.

\section{Trigonometric Function Solutions}

When $\lambda^{2}-4 \mu<0$, we obtain

$$
v(\xi)=-\frac{4 \mu-\lambda^{2}}{2}\left(\frac{-C_{1} \sin (1 / 2) \sqrt{4 \mu-\lambda^{2}} \xi+C_{2} \cos (1 / 2) \sqrt{4 \mu-\lambda^{2}} \xi}{C_{1} \cos (1 / 2) \sqrt{4 \mu-\lambda^{2}} \xi+C_{2} \sin (1 / 2) \sqrt{4 \mu-\lambda^{2}} \xi}\right)^{2}+\frac{1}{3} \lambda^{2}-\frac{4}{3} \mu,
$$

where $\xi=x+\left(-\lambda^{4}+8 \mu \lambda^{2}-16 \mu^{2}\right) t, C_{1}$ and $C_{2}$ are arbitrary constants.

The various known results can be rediscovered, if $C_{1}$ and $C_{2}$ are taken as special values. 


\section{Rational Function Solution}

When $\lambda^{2}-4 \mu=0$, we obtain

$$
v(\xi)=-\frac{2 C_{2}^{2}}{\left(C_{1}+C_{2} \xi\right)^{2}}+\frac{1}{3} \lambda^{2}-\frac{4}{3} \mu,
$$

where $\xi=x+\left(-\lambda^{4}+8 \mu \lambda^{2}-16 \mu^{2}\right) t, C_{1}$, and $C_{2}$ are arbitrary constants.

Case 2. Substituting (3.7) into (3.4) yields

$$
v(\xi)=-\left(\frac{G^{\prime}}{G}\right)^{2}-\lambda\left(\frac{G^{\prime}}{G}\right)+\alpha_{0}
$$

Substituting the general solution of (2.5) into (3.15), we obtain three types of traveling wave solutions of (3.3) as follows.

Hyperbolic Function Solutions

When $\lambda^{2}-4 \mu>0$, we obtain

$$
v(\xi)=-\frac{\lambda^{2}-4 \mu}{2}\left(\frac{C_{1} \sinh (1 / 2) \sqrt{\lambda^{2}-4 \mu} \xi+C_{2} \cosh (1 / 2) \sqrt{\lambda^{2}-4 \mu} \xi}{C_{1} \cosh (1 / 2) \sqrt{\lambda^{2}-4 \mu} \xi+C_{2} \sinh (1 / 2) \sqrt{\lambda^{2}-4 \mu} \xi}\right)^{2}+\frac{1}{4} \lambda^{2}+\alpha_{0},
$$

where $\xi=x+\left(-180 \alpha_{0}^{2}-30 \lambda^{2} \alpha_{0}-22 \mu \lambda^{2}-76 \mu^{2}-\lambda^{4}-240 \mu \alpha_{0}\right) t$. $C_{1}$ and $C_{2}$ are arbitrary constants.

The various known results can be rediscovered, if $C_{1}$ and $C_{2}$ are taken as special values.

\section{Trigonometric Function Solutions}

When $\lambda^{2}-4 \mu<0$, we obtain

$$
v(\xi)=-\frac{4 \mu-\lambda^{2}}{4}\left(\frac{-C_{1} \sin (1 / 2) \sqrt{4 \mu-\lambda^{2}} \xi+C_{2} \cos (1 / 2) \sqrt{4 \mu-\lambda^{2}} \xi}{C_{1} \cos (1 / 2) \sqrt{4 \mu-\lambda^{2}} \xi+C_{2} \sin (1 / 2) \sqrt{4 \mu-\lambda^{2}} \xi}\right)^{2}+\frac{1}{4} \lambda^{2}+\alpha_{0},
$$

where $\xi=x+\left(-180 \alpha_{0}^{2}-30 \lambda^{2} \alpha_{0}-22 \mu \lambda^{2}-76 \mu^{2}-\lambda^{4}-240 \mu \alpha_{0}\right) t$. $C_{1}$ and $C_{2}$ are arbitrary constants. The various known results can be rediscovered, if $C_{1}$ and $C_{2}$ are taken as special values. For example:

(i) if $C_{1}=0$ but $C_{2} \neq 0$, we obtain

$$
v(\xi)=-\frac{4 \mu-\lambda^{2}}{4} \cot ^{2} \frac{1}{2} \sqrt{4 \mu-\lambda^{2}} \xi+\frac{1}{4} \lambda^{2}+\alpha_{0}
$$




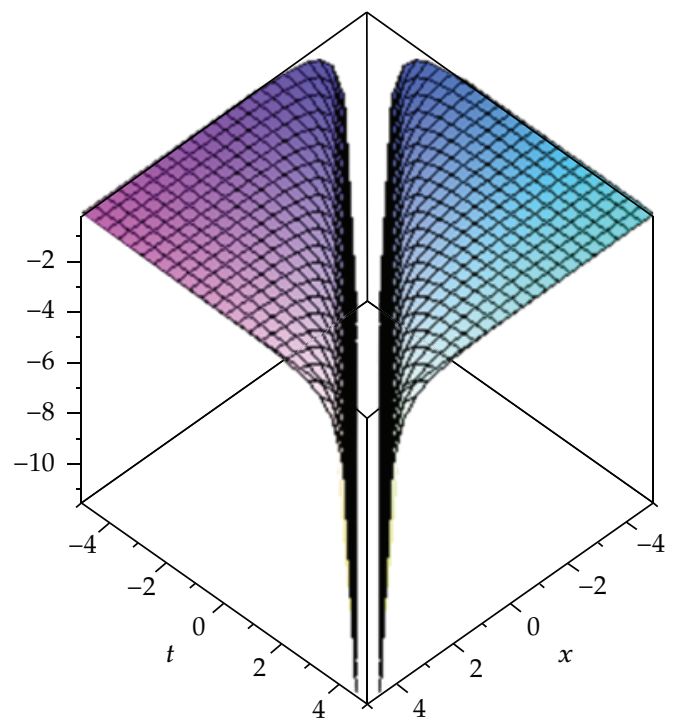

Figure 1: Solitary wave obtained from solution (3.10), for $\lambda=3, \mu=2$.

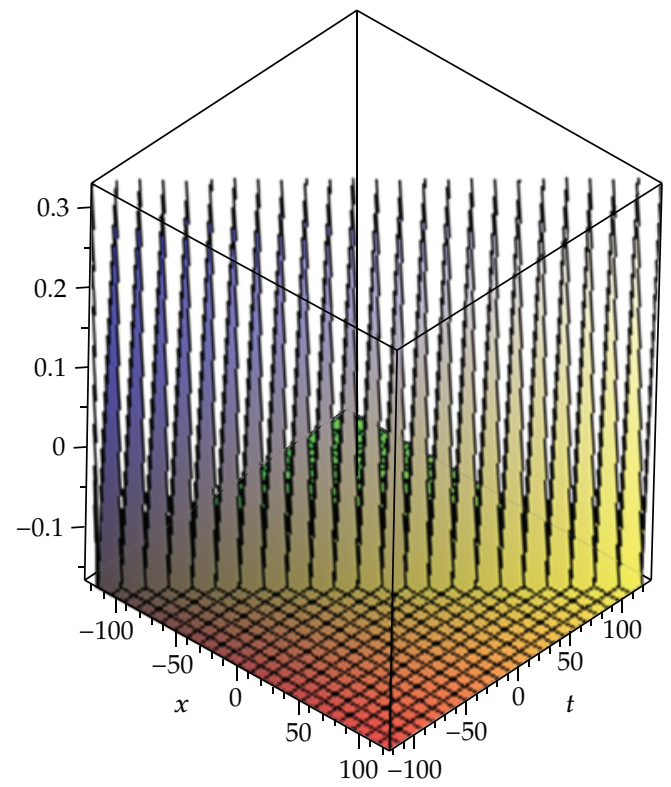

Figure 2: Solitary wave obtained from solution (3.11), for $\lambda=3, \mu=2$.

(ii) If $C_{2}=0$ but $C_{1} \neq 0$, we obtain

$$
v(\xi)=\frac{\lambda^{2}-4 \mu}{4} \tan ^{2} \frac{1}{2} \sqrt{4 \mu-\lambda^{2}} \xi+\frac{1}{4} \lambda^{2}+\alpha_{0} .
$$




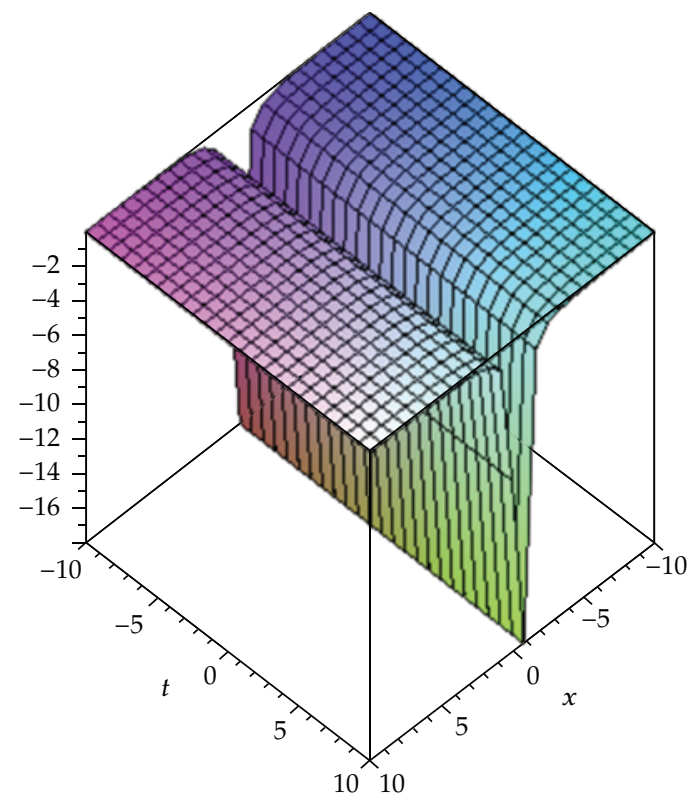

Figure 3: Solitary wave obtained from solution (3.14), for $\lambda=2, \mu=1, C_{1}=1, C_{2}=2$.

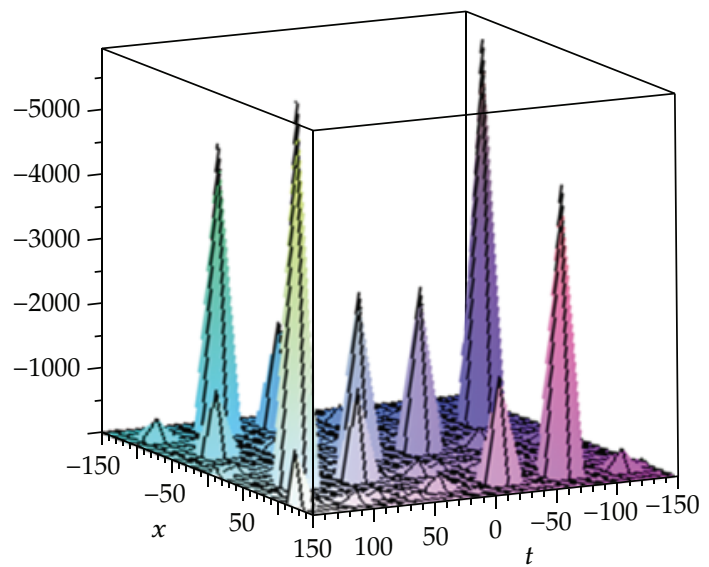

Figure 4: Solitary wave obtained from solution (3.19), for $\lambda=1, \mu=1, \alpha_{0}=1$.

(iii) If $C_{1} \neq 0$ and $C_{1}^{2}>C_{2}^{2}$, we obtain

$$
v(\xi)=-\frac{4 \mu-\lambda^{2}}{4} \cot ^{2}\left(\frac{1}{2} \sqrt{4 \mu-\lambda^{2}} \xi+\xi_{0}\right)+\frac{1}{4} \lambda^{2}+\alpha_{0}
$$

where $\xi_{0}=\tan ^{-1} C_{2} / C_{1}$. 


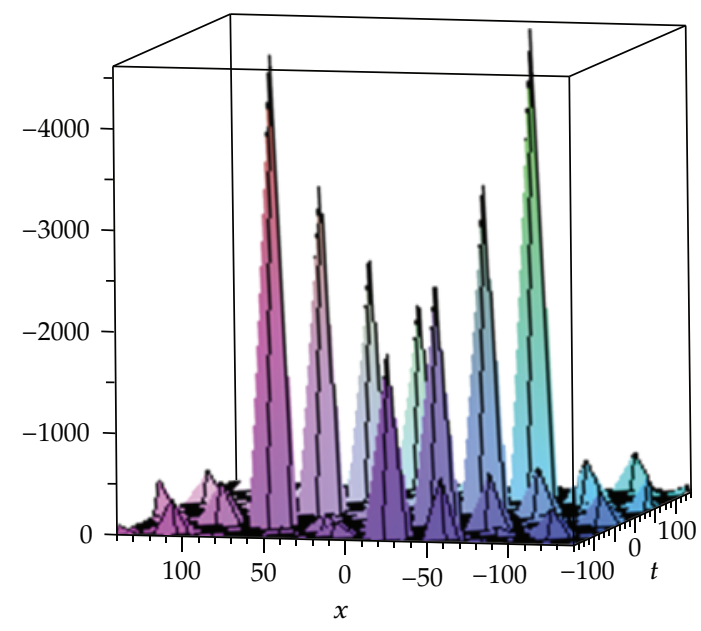

Figure 5: Solitary wave obtained from solution (3.19), for $\lambda=1, \mu=1, \alpha_{0}=1$.

\section{Rational Function Solution}

When $\lambda^{2}-4 \mu=0$,

$$
v(\xi)=-\frac{C_{2}^{2}}{\left(C_{1}+C_{2} \xi\right)^{2}}+\frac{1}{4} \lambda^{2}+\alpha_{0}
$$

where $\xi=x+\left(-180 \alpha_{0}^{2}-30 \lambda^{2} \alpha_{0}-22 \mu \lambda^{2}-76 \mu^{2}-\lambda^{4}-240 \mu \alpha_{0}\right) t . C_{1}$ and $C_{2}$ are arbitrary constants.

\subsection{Discussion}

The solutions of the CDG (3.1) are investigated by different methods, such as Jin [32] investigated solutions by the variational iteration method, Salas [33] by the projective Riccati equation method, and Wazwaz [34] by using the tanh method. To the best of our awareness the CDG equation is not solved by the prominent $\left(G^{\prime} / G\right)$-expansion method. In this paper, we solve this equation by the $\left(G^{\prime} / G\right)$-expansion method. It is noteworthy to point out that our attained solutions are new and cannot be found from above author's solutions by any choice of arbitrary constants.

\subsection{Graphical Representations of the Solutions}

The solutions are shown in the graphs with the aid of Maple 13 in Figures 1-5.

\section{Conclusions}

In this paper, three types of traveling wave solutions, such as the hyperbolic, the trigonometric, and the rational functions of the Caudrey-Dodd-Gibbon equation are successfully obtained by using the $\left(G^{\prime} / G\right)$-expansion method. Exact traveling wave solutions of this equation have many potential applications in engineering and mathematical physics. 
The obtained solutions also show that the method is effective, more powerful, and simple for searching exact traveling wave solutions of the NLPDEs. The method can be applied in different types of NLEEs and it is our task in the future.

\section{Acknowledgments}

This paper is supported by USM short term Grant (no. 304/PMATHS/6310072). The authors would like to thank the School of Mathematical Sciences, USM, for providing related research facilities.

\section{References}

[1] L. Jianming, D. Jie, and Y. Wenjun, "Backlund transformation and new exact solutions of the SharmaTasso-Olver equation," Abstract and Applied Analysis, Article ID 935710, 8 pages, 2011.

[2] K. Al-Khaled, M. Al-Refai, and A. Alawneh, "Traveling wave solutions using the variational method and the tanh method for nonlinear coupled equations," Applied Mathematics and Computation, vol. 202, no. 1, pp. 233-242, 2008.

[3] A. Bekir, "Applications of the extended tanh method for coupled nonlinear evolution equations," Communications in Nonlinear Science and Numerical Simulation, vol. 13, no. 9, pp. 1748-1757, 2008.

[4] S. A. Yousefi, A. Lotfi, and M. Dehghan, "He's variational iteration method for solving nonlinear mixed volterra-fredholm integral equations," Computers $\mathcal{E}$ Mathematics with Applications, vol. 58, no. 11-12, pp. 2172-2176, 2009.

[5] R. Rajaram and M. Najafi, "Analytical treatment and convergence of the adomian decomposition method for a system of coupled damped wave equations," Applied Mathematics and Computation, vol. 212, no. 1, pp. 72-81, 2009.

[6] J. L. Li, “Adomian's decomposition method and homotopy perturbation method in solving nonlinear equations," Journal of Computational and Applied Mathematics, vol. 228, no. 1, pp. 168-173, 2009.

[7] E. Hesameddini and A. Peyrovi, "Homotopy perturbation method for second painleve equation and comparisons with analytic continuation extension and chebishev series method," Journal for Theory and Applications, vol. 5, no. 13, pp. 629-637, 2010.

[8] E. S. A. Alaidarous, "F-expansion method for the nonlinear generalized Ito system," International Journal of Basic E Applied Sciences, vol. 10, no. 2, pp. 90-117, 2010.

[9] Z. J. Zhou, J. Z. Fu, and Z. B. Li, "Maple packages for computing Hirota's bilinear equation and multisoliton solutions of nonlinear evolution equations," Applied Mathematics and Computation, vol. 217, no. 1, pp. 92-104, 2010.

[10] A. Bekir and E. Aksoy, "Exact solutions of nonlinear evolution equations with variable coefficients using exp-function method," Applied Mathematics and Computation, vol. 217, no. 1, pp. 430-436, 2010.

[11] A. H. Salas and C. A. Gomez, "Application of the Cole-Hopf transformation for finding exact solutions to several forms of the seventh-order KdV equation," Mathematical Problems in Engineering, Article ID 194329, 14 pages, 2010.

[12] A. H. Salas and C. A. Gomez, "Exact solutions for a third-order KdV equation with variable coefficients and forcing term," Mathematical Problems in Engineering, Article ID 737928, 13 pages, 2009.

[13] M. A. Noor and S. T. Mohyud-Din, "Modified variational iteration method for goursat and laplace problems," World Applied Sciences Journal, vol. 4, no. 4, pp. 487-498, 2008.

[14] W. X. Ma and J. H. Lee, "A transformed rational function method and exact solutions to the $3+1$ dimensional Jimbo-Miwa equation," Chaos, Solitons and Fractals, vol. 42, no. 3, pp. 1356-1363, 2009.

[15] Y. Khan, N. Faraz, and A. Yildirim, "New soliton solutions of the generalized Zakharov equations using he's variational approach," Applied Mathematics Letters, vol. 24, no. 6, pp. 965-968, 2011.

[16] S. A. El-Wakil, A. R. Degheidy, E. M. Abulwafa, M. A. Madkour, M. T. Attia, and M. A. Abdou, "Exact travelling wave solutions of generalized Zakharov equations with arbitrary power nonlinearities," International Journal of Nonlinear Science, vol. 7, no. 4, pp. 455-461, 2009.

[17] S. M. Sayed, O. O. Elhamahmy, and G. M. Gharib, "Travelling wave solutions for the KdV-BurgersKuramoto and nonlinear Schrodinger equations which describe pseudospherical surfaces," Journal of Applied Mathematics, Article ID 576783, 10 pages, 2008.

[18] J. Zhou, L. Tian, and X. Fan, "Soliton and periodic wave solutions to the osmosis $(2,2)$ equation," Mathematical Problems in Engineering, Article ID 509390, 10 pages, 2009. 
[19] M. A. Noor, K. I. Noor, E. Al-Said, and M. Waseem, "Some new iterative methods for nonlinear equations," Mathematical Problems in Engineering, Article ID 198943, 12 pages, 2010.

[20] C. A. Gomez and A. H. Salas, "Exact solutions for the generalized BBM equation with variable coefficients," Mathematical Problems in Engineering, Article ID 498249, 10 pages, 2010.

[21] M. Wang, X. Li, and J. Zhang, "The $\left(G^{\prime} / G\right)$-expansion method and travelling wave solutions of nonlinear evolution equations in mathematical physics," Physics Letters A, vol. 372, no. 4, pp. 417$423,2008$.

[22] R. Abazari, "Application of $\left(G^{\prime} / G\right)$-expansion method to travelling wave solutions of three nonlinear evolution equation," Computers \& Fluids, vol. 39, no. 10, pp. 1957-1963, 2010.

[23] B. Zheng, "Travelling wave solutions of two nonlinear evolution equations by using the $\left(G^{\prime} / G\right)$ expansion method," Applied Mathematics and Computation, vol. 217, no. 12, pp. 5743-5753, 2011.

[24] J. Feng, W. Li, and Q. Wan, “Using $\left(G^{\prime} / G\right)$-expansion method to seek the traveling wave solution of Kolmogorov-Petrovskii-Piskunov equation," Applied Mathematics and Computation, vol. 217, no. 12, pp. 5860-5865, 2011.

[25] X. Liu, L. Tian, and Y. Wu, "Application of $\left(G^{\prime} / G\right)$-expansion method to two nonlinear evolution equations," Applied Mathematics and Computation, vol. 217, no. 4, pp. 1376-1384, 2010.

[26] J. Feng, "New traveling wave solutions to the seventh-order Sawada-Kotera equation," Journal of Applied Mathematics E Informatics, vol. 28, no. 5-6, pp. 1431-1437, 2010.

[27] S. Guo and Y. Zhou, "The extended $\left(G^{\prime} / G\right)$-expansion method and its applications to the WhithamBroer-Kaup-like equations and coupled Hirota-Satsuma KdV equations," Applied Mathematics and Computation, vol. 215, no. 9, pp. 3214-3221, 2010.

[28] E. M. E. Zayed and S. Al-Joudi, "Applications of an extended ( $\left.G^{\prime} / G\right)$-expansion method to find exact solutions of nonlinear PDEs in mathematical physics," Mathematical Problems in Engineering, vol. 2010, Article ID 768573, 19 pages, 2010.

[29] J. Zhang, F. Jiang, and X. Zhao, "An improved $\left(G^{\prime} / G\right)$-expansion method for solving nonlinear evolution equations," International Journal of Computer Mathematics, vol. 87, no. 8, pp. 1716-1725, 2010.

[30] L. Li, E. Li, and M. Wang, "The $\left(G^{\prime} / G, 1 / G\right)$-expansion method and its application to travelling wave solutions of the Zakharov equations," Applied Mathematics B, vol. 25, no. 4, pp. 454-462, 2010.

[31] M. Hayek, "Constructing of exact solutions to the KdV and Burgers equations with power-law nonlinearity by the extended $\left(G^{\prime} / G\right)$-expansion method," Applied Mathematics and Computation, vol. 217 , no. 1, pp. 212-221, 2010.

[32] L. Jin, "Application of the variational iteration method for solving the fifth order caudrey-doddgibbon equation," International Mathematical Forum, vol. 5, no. 66, pp. 3259-3265, 2010

[33] A. Salas, "Some exact solutions for the caudrey-dodd-gibbon equation," Mathematical Physics, 2008, arXiv:0805.2969v2.

[34] A. M. Wazwaz, "Analytic study of the fifth order integrable nonlinear evolution equations by using the tanh method," Applied Mathematics and Computation, vol. 174, no. 1, pp. 289-299, 2006. 


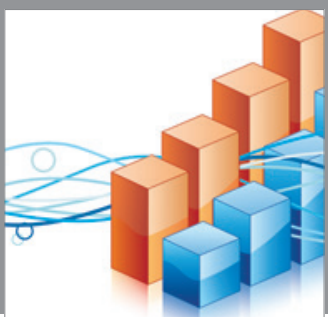

Advances in

Operations Research

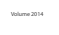

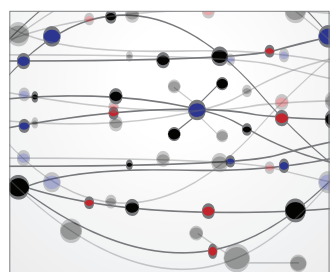

\section{The Scientific} World Journal
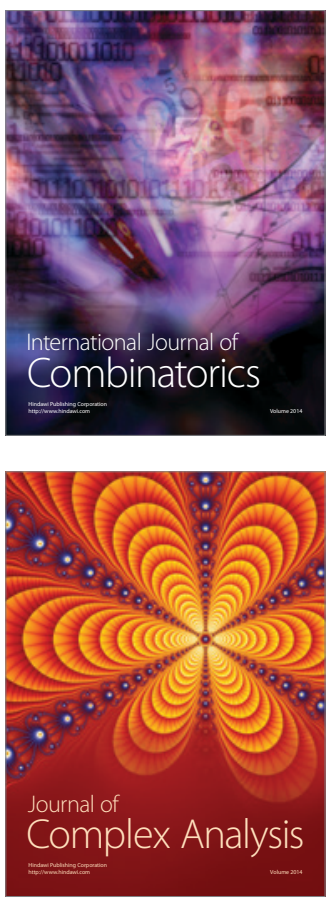

International Journal of

Mathematics and

Mathematical

Sciences
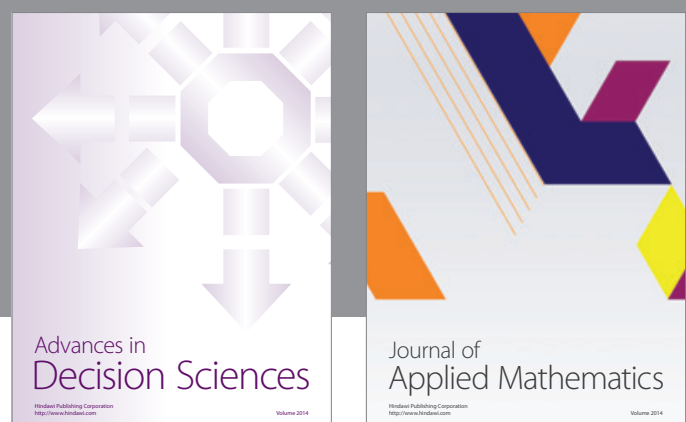

Journal of

Applied Mathematics
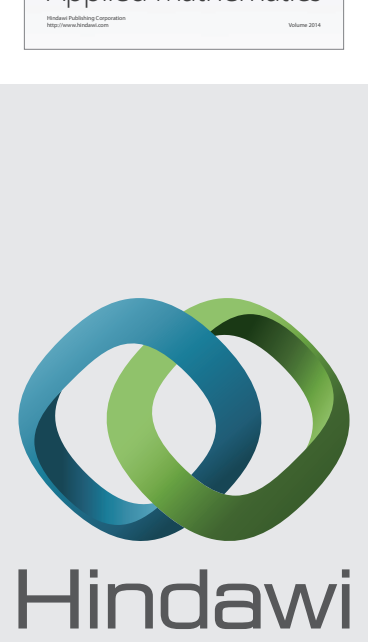

Submit your manuscripts at http://www.hindawi.com
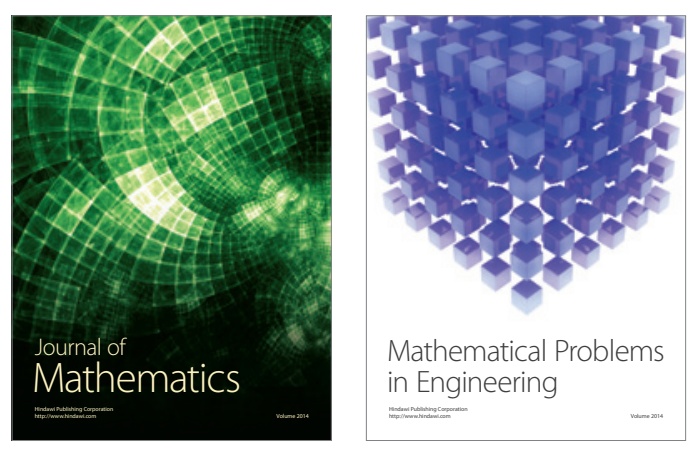

Mathematical Problems in Engineering
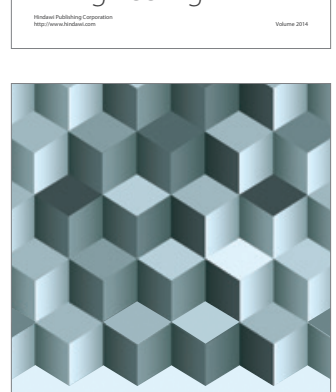

Journal of

Function Spaces
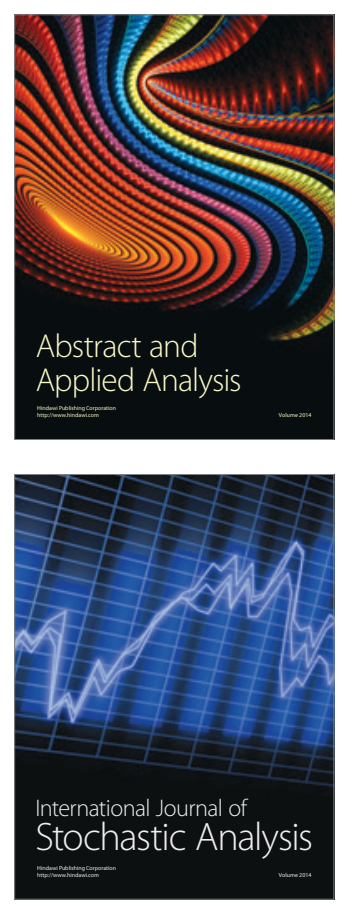

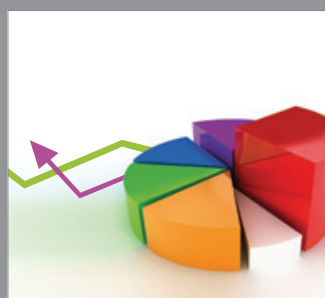

ournal of

Probability and Statistics

Promensencen
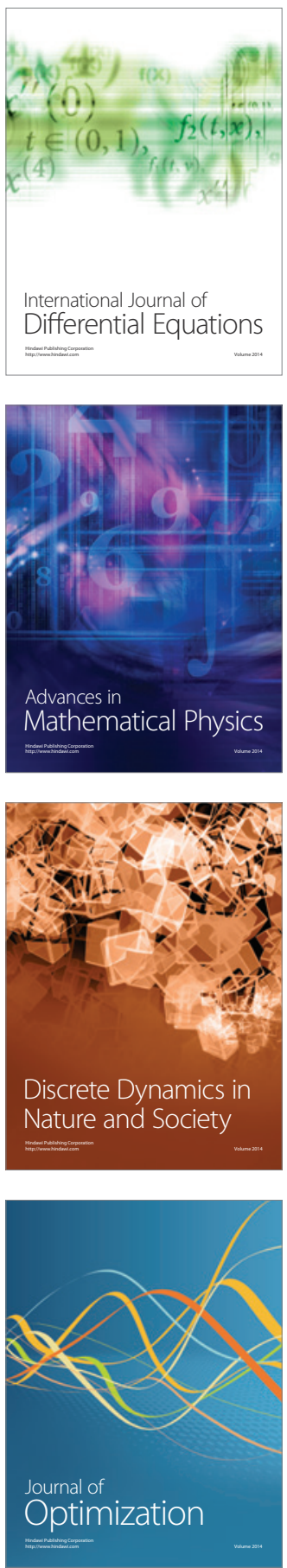